\title{
SISTEMAS DE GRAMÁTICAS Y PROCESAMIENTO MODULAR
}

\section{Carlos Martín Vide}

\begin{abstract}
The new mathematical theory of grammar systems is an example of multi-agent system trying to formally model the cooperative interaction of several processors towards the common aim to generate/accept a language. Given the fact that natural languages seem to be modular structures, the theory of grammar systems appears as a good candidate to adequately give account of them. Grammar systems allow to generate a non-context-free language by means of only regular rules, which is a spectacular feature from the viewpoint of classical formal language theory. Moreover, grammar systems show advantages in terms of computational complexity. We introduce here in the theory.
\end{abstract}

\section{Idea de un sistema de gramáticas}

Sabemos que en la teoría clásica de lenguajes formales cada gramática y cada autómata operan individualmente, de manera que una gramática [respectivamente, un autómata] genera [respectivamente, reconoce] un lenguaje. Hoy, sin embargo, conceptos como distribución, cooperación, paralelismo, comunicación van ganando terreno progresivamente en diferentes ámbitos de las ciencias de la computación. Parece incluso bastante razonable que el cerebro humano funcione de una manera distribuida, sobre todo a la vista de la gran cantidad de capacidades y habilidades que parece poner en juego simultáneamente y de la plausibilidad de que tenga una estructura modular. Los sistemas de gramáticas constituyen un modelo matemático del procesamiento tanto distribuido como paralelo de la información.

Un sistema de gramáticas es una estructura compuesta por diversas gramáticas que funcionan coordinadas, conforme a un protocolo o algoritmo especificado, para producir un lenguaje. El citado protocolo de cooperación es el elemento crucial. Esta maquinaria generativa tiene especial flexibilidad para alcanzar objetivos tales como:

a) modelizar un fenómeno real complejo,

b) afinar la capacidad generativa,

c) disminuir la complejidad descriptiva.

Las clases de sistemas de gramáticas que vamos a considerar aquí son las siguientes:

1) sistemas con componentes que funcionan secuencialmente, estando activo uno en cada momento ("cooperating distributed grammar systems", o sistemas de 
gramáticas distribuidas en cooperación, introducidos en Csuhaj-Varjú \& Dassow 1989, a partir de las ideas de Meersman \& Rozenberg 1978), y

2) sistemas con componentes que funcionan en paralelo, sincrónicamente ("parallel communicating grammar systems", o sistemas de gramáticas comunicadas en paralelo, introducidos en Păun \& Sântean 1989).

Las referencias más accesibles y actuales para iniciarse en la materia son Csuhaj-Varjú, Dassow, Kelemen \& Păun 1994, Păun 1995a y Păun 1995c).

En un sistema de gramáticas distribuidas en cooperación, diversas gramáticas trabajan juntas, sucesivamente, sobre un axioma común. En cada paso de la derivación está activa una gramática, y las demás permanecen inactivas. Las condiciones bajo las cuales una gramática puede activarse o desactivarse aparecen especificadas en el protocolo de cooperación. Cada gramática ha de usar un cierto número de reglas exactamente $(=\mathrm{k})$, como mínimo ese número $(\geq \mathrm{k})$, como máximo ese número $(\leq \mathrm{k})$, un número cualquiera $\left(^{*}\right)$ o el máximo número posible de reglas (t). El lenguaje compuesto por las cadenas terminales así generadas es el lenguaje generado por el sistema.

En un sistema de gramáticas comunicadas en paralelo, cada gramática trabaja sobre su propio axioma al mismo tiempo que las restantes trabajan sobre sus axiomas respectivos, empleando cada una de ellas una regla en cada unidad de tiempo. Hasta aquí, se trata de gramáticas separadas que funcionan separadamente. Lo que da nacimiento a un sistema es la posibilidad de comunicación. Cuando un componente del sistema introduce un símbolo de llamada, se desencadena la comunicación. Una de las gramáticas se encarga de la dirección del proceso. El lenguaje generado por ésta es el lenguaje que el sistema produce.

Las convenciones notacionales básicas que utilizaremos son las usuales: $\mathrm{V}^{*}$ es el monoide libre generado por el alfabeto $\mathrm{V}$ con la operación de concatenación, $\lambda$ es la cadena vacía, $\mathrm{V}^{+}=\mathrm{V}^{*}-\{\lambda\},|\mathrm{x}|$ es la longitud de $\mathrm{x} \in \mathrm{V}^{*}$ y $|\mathrm{x}|_{\mathrm{U}}$ es el número de ocurrencias de símbolos de $U \subseteq V^{*}$ en $x$. Una gramática de Chomsky es una estructura $G=\left(V_{N}, V_{T}, S, P\right)$, donde $\mathrm{V}_{\mathrm{N}}$ es el alfabeto no-terminal, $\mathrm{V}_{\mathrm{T}}$ el alfabeto terminal, $\mathrm{S}$ el axioma y $\mathrm{P}$ el conjunto de producciones. Una de las referencias clásicas en la teoría de lenguajes formales, a la que nos remitimos, es Salomaa (1973).

\section{Sistemas de gramáticas distribuidas en cooperación}

Definición 1. Un sistema de gramáticas distribuidas en cooperación (CD) es una estructura:

$$
\Gamma=\left(\mathrm{V}_{\mathrm{N}}, \mathrm{V}_{\mathrm{T}}, \mathrm{S}, \mathrm{P}_{1}, \ldots, \mathrm{P}_{\mathrm{n}}\right),
$$

con $\mathrm{n} \geq 1$, donde $\mathrm{V}_{\mathrm{N}} \cap \mathrm{V}_{\mathrm{T}}=\varnothing, \mathrm{S} \in \mathrm{V}_{\mathrm{N}}$ y $\mathrm{P}_{1}, \ldots, \mathrm{P}_{\mathrm{n}}$ son conjuntos finitos de reglas de reescritura (usualmente independientes del contexto, aunque cabe considerar cualquier otro tipo de reglas pertenecientes a cualquier clase de mecanismo generativo) sobre $\mathrm{V}_{\mathrm{N}} \cup \mathrm{V}_{\mathrm{T}}$, a los que llamamos componentes del sistema.

Definición 2. En $\left(\mathrm{V}_{\mathrm{N}} \cup \mathrm{V}_{\mathrm{T}}\right)^{*}$ se define la derivación inmediata con respecto a $\mathrm{P}_{\mathrm{i}}$, con $1 \leq \mathrm{i} \leq \mathrm{n}$, del modo usual, y se nota $\Rightarrow_{\mathrm{i}}$. Las derivaciones que constan de exactamente $\mathrm{k}$, a lo sumo $\mathrm{k}$ y al menos $\mathrm{k}$ pasos se notan, respectivamente, con $\Rightarrow_{\mathrm{i}}{ }^{\mathrm{k}}, \Rightarrow_{\mathrm{i}} \mathrm{k} \mathrm{y} \Rightarrow_{\mathrm{i}}^{\mathrm{k}}$, con $\mathrm{k} \geq 1$. Una 
derivación arbitraria se expresa como $\Rightarrow_{\mathrm{i}}{ }^{*}$, y una derivación máxima o terminal como $\Rightarrow_{\mathrm{i}}^{\mathrm{t}}$. Esta última circunstancia se caracteriza formalmente así:

$$
\mathrm{x} \Rightarrow_{\mathrm{i}}^{\mathrm{t}} \mathrm{y} \operatorname{ssi}\left(\mathrm{x} \Rightarrow_{\mathrm{i}}^{\geq 1} \mathrm{y} \wedge \neg \exists \mathrm{z} \in\left(\mathrm{V}_{\mathrm{N}} \cup \mathrm{V}_{\mathrm{T}}\right)^{*} \mid \mathrm{y} \Rightarrow_{\mathrm{i}} \mathrm{z}\right) .
$$

Definición 3. El lenguaje generado por $\Gamma$ en el modo $g \in\{\leq k,=k, \geq k \mid k \geq 1\} \cup\{*, t\}$ se define como:

$$
\mathrm{L}_{\mathrm{g}}(\Gamma)=\left\{\mathrm{x} \in \mathrm{V}_{\mathrm{T}} * \mid \mathrm{S} \Rightarrow \mathrm{i}_{1}{ }^{\mathrm{g}} \mathrm{x}_{1} \Rightarrow \mathrm{i}_{2}{ }^{\mathrm{g}} \ldots \Rightarrow \mathrm{i}_{\mathrm{m}}{ }^{\mathrm{g}} \mathrm{x}_{\mathrm{m}}=\mathrm{x} \text {, con } \mathrm{m} \geq 1,1 \leq \mathrm{i}_{\mathrm{j}} \leq \mathrm{n}, 1 \leq \mathrm{j} \leq \mathrm{m}\right\} .
$$

Ejemplo 4. Considérese el siguiente sistema $\mathrm{CD}$ :

$$
\Gamma=\left(\left\{\mathrm{S}, \mathrm{A}, \mathrm{A}^{\prime}, \mathrm{B}, \mathrm{B}^{\prime}\right\},\{\mathrm{a}, \mathrm{b}, \mathrm{c}\}, \mathrm{S}, \mathrm{P}_{1}, \mathrm{P}_{2}\right),
$$

con:

$$
\begin{gathered}
P_{1}=\left\{S \rightarrow S, S \rightarrow A B, A^{\prime} \rightarrow A, B^{\prime} \rightarrow B\right\}, \\
P_{2}=\left\{A \rightarrow a A^{\prime} b, B \rightarrow c B^{\prime}, A \rightarrow a b, B \rightarrow c\right\} .
\end{gathered}
$$

Dependiendo del modo elegido de derivación, $\Gamma$ genera, entre otros, los siguientes lenguajes:

$$
\begin{aligned}
& \mathrm{L}_{\mathrm{g}}(\Gamma)=\left\{\mathrm{a}^{\mathrm{n}} \mathrm{b}^{\mathrm{n}} \mathrm{c}^{\mathrm{n}} \mid \mathrm{n} \geq 1\right\}, \text { con } \mathrm{g} \in\{=2, \geq 2\}, \\
& \mathrm{L}_{\mathrm{g}}(\Gamma)=\left\{\mathrm{a}^{\mathrm{n}} \mathrm{b}^{\mathrm{n}} \mathrm{c}^{\mathrm{m}} \mid \mathrm{n}, \mathrm{m} \geq 1\right\}, \text { con } \mathrm{g} \in\{=1, \geq 1, *, \mathrm{t}\} \cup\{\leq \mathrm{k} \mid \mathrm{k} \geq 1\}, \\
& \mathrm{L}_{\mathrm{g}}(\Gamma)=\varnothing, \text { con } \mathrm{g} \in\{=\mathrm{k}, \geq \mathrm{k} \mid \mathrm{k} \geq 3\} .
\end{aligned}
$$

Veamos en detalle cómo funciona $\Gamma$ en el modo $=2$. Hemos de empezar por S. Sólo se puede usar $P_{1}$. Aplicar dos veces la regla $S \rightarrow S$ no cambia nada, de modo que optamos por usar dos reglas diferentes:

$$
\mathrm{S} \Rightarrow_{1} \mathrm{~S} \Rightarrow_{1} \mathrm{AB} \text {. }
$$

De ahora en adelante ya no volverá a aparecer S. A AB únicamente se le puede aplicar $\mathrm{P}_{2}$. Si usamos las reglas no-terminales, obtenemos:

$$
\mathrm{AB} \Rightarrow_{2} \mathrm{aA} \mathrm{A}^{\prime} \mathrm{bB} \Rightarrow_{2} \mathrm{aA}^{\prime} \mathrm{bcB} \text {. }
$$

Ahora sólo podemos emplear $\mathrm{P}_{1}$ :

$$
\mathrm{aA}{ }^{\prime} \mathrm{bcB}^{\prime} \Rightarrow_{1} \mathrm{aAbcB}{ }^{\prime} \Rightarrow_{1} \mathrm{aAbcB} \text {. }
$$

A continuación, de nuevo $\mathrm{P}_{2}$ :

$$
\mathrm{aAbcB} \Rightarrow_{2} \text { aaA'bbcB } \Rightarrow_{2} \text { aaA'bbccB'. }
$$

Volvemos a $\mathrm{P}_{1}$, para obtener:

$$
\text { aaA'bbccB' } \Rightarrow_{1} \text { aaAbbccB' } \Rightarrow_{1} \text { aaAbbccB. }
$$

Si queremos terminar, hemos de utilizar otra vez $\mathrm{P}_{2}$ :

$$
\text { aaAbbccB } \Rightarrow_{2} \text { aaabbbccc. }
$$

Así pues, hemos de usar sucesivamente $\mathrm{P}_{1}$ y las reglas no-terminales de $\mathrm{P}_{2}$, y acabamos la derivación con las reglas terminales de $\mathrm{P}_{2}$. En conclusión, en efecto:

$$
\mathrm{L}_{=2}(\Gamma)=\left\{\mathrm{a}^{\mathrm{n}} \mathrm{b}^{\mathrm{n}} \mathrm{c}^{\mathrm{n}} \mid \mathrm{n} \geq 1\right\} .
$$


Ejemplo 5. El sistema de gramáticas $\mathrm{CD}$ :

$$
\Gamma=\left(\{\mathrm{S}, \mathrm{A}\},\{\mathrm{a}\}, \mathrm{S}, \mathrm{P}_{1}, \mathrm{P}_{2}, \mathrm{P}_{3}\right),
$$

con:

$$
\begin{gathered}
P_{1}=\{S \rightarrow A A\}, \\
P_{2}=\{A \rightarrow S\}, \\
P_{3}=\{S \rightarrow a\},
\end{gathered}
$$

genera el lenguaje:

$$
\mathrm{L}_{\mathrm{t}}(\Gamma)=\left\{\mathrm{a}^{2 \mathrm{n}} \mid \mathrm{n} \geq 0\right\} .
$$

Ejemplo 6. El sistema de gramáticas $\mathrm{CD}$ :

$$
\Gamma=\left(\left\{\mathrm{S}, \mathrm{A}, \mathrm{A}^{\prime}\right\},\{\mathrm{a}, \mathrm{b}\}, \mathrm{S}, \mathrm{P}_{1}, \mathrm{P}_{2}, \mathrm{P}_{3}\right),
$$

con:

$$
\begin{aligned}
\mathrm{P}_{1}= & \left\{\mathrm{S} \rightarrow \mathrm{S}, \mathrm{S} \rightarrow \mathrm{AA}, \mathrm{A}^{\prime} \rightarrow \mathrm{A}\right\}, \\
\mathrm{P}_{2} & =\left\{\mathrm{A} \rightarrow \mathrm{aA} A^{\prime}, \mathrm{A} \rightarrow \mathrm{a}\right\}, \\
\mathrm{P}_{3} & =\left\{\mathrm{A} \rightarrow \mathrm{bA}^{\prime}, \mathrm{A} \rightarrow \mathrm{b}\right\},
\end{aligned}
$$

genera el lenguaje:

$$
\mathrm{L}_{=2}(\Gamma)=\mathrm{L}_{22}(\Gamma)=\left\{\mathrm{xx} \mid \mathrm{x} \in\{\mathrm{a}, \mathrm{b}\}^{+}\right\} .
$$

\section{Sistemas de gramáticas comunicadas en paralelo}

Definición 7. Un sistema de gramáticas comunicadas en paralelo (PC) es una estructura:

$$
\Gamma=\left(\mathrm{V}_{\mathrm{N}}, \mathrm{K}, \mathrm{V}_{\mathrm{T}},\left(\mathrm{S}_{1}, \mathrm{P}_{1}\right), \ldots,\left(\mathrm{S}_{\mathrm{n}}, \mathrm{P}_{\mathrm{n}}\right)\right)
$$

donde $\mathrm{V}_{\mathrm{N}}, \mathrm{K}, \mathrm{V}_{\mathrm{T}}$ son alfabetos disjuntos, con $\mathrm{K}=\left\{\mathrm{Q}_{1}, \ldots, \mathrm{Q}_{\mathrm{n}}\right\}, \mathrm{S}_{\mathrm{i}} \in \mathrm{V}_{\mathrm{N}}$ y $\mathrm{P}_{\mathrm{i}}$ son conjuntos finitos de reglas de reescritura (usualmente independientes del contexto, aunque cabe considerar cualquier otro tipo de reglas pertenecientes a cualquier clase de mecanismo generativo) sobre $V_{\Gamma}=V_{N} \cup K \cup V_{T}$, con $1 \leq i \leq n$. Los elementos de $\mathrm{K}$ reciben el nombre de símbolos de llamada, $\left(\mathrm{S}_{\mathrm{i}}, \mathrm{P}_{\mathrm{i}}\right)$ son los componentes de $\Gamma$, con $1 \leq \mathrm{i} \leq \mathrm{n}$, y existe una correspondencia biyectiva entre los símbolos de llamada y los componentes, de tal manera que cada símbolo de llamada hace referencia al componente cuyo subíndice es el mismo. Una n-upla $\left(\mathrm{x}_{1}, \ldots, \mathrm{x}_{\mathrm{n}}\right)$, con $\mathrm{x}_{\mathrm{i}} \in \mathrm{V}_{\Gamma}{ }^{*}$ y $1 \leq \mathrm{i} \leq \mathrm{n}$ es una configuración de $\Gamma$.

Definición 8. Dado un sistema de gramáticas $\mathrm{PC}$ :

$$
\Gamma=\left(\mathrm{V}_{\mathrm{N}}, \mathrm{K}, \mathrm{V}_{\mathrm{T}},\left(\mathrm{S}_{1}, \mathrm{P}_{1}\right), \ldots,\left(\mathrm{S}_{\mathrm{n}}, \mathrm{P}_{\mathrm{n}}\right)\right),
$$

y dadas dos configuraciones:

$$
\begin{aligned}
& \left(\mathrm{x}_{1}, \ldots, \mathrm{x}_{\mathrm{n}}\right), \\
& \left(\mathrm{y}_{1}, \ldots, \mathrm{y}_{\mathrm{n}}\right),
\end{aligned}
$$

con $\mathrm{x}_{\mathrm{i}}, \mathrm{y}_{\mathrm{i}} \in \mathrm{V}_{\Gamma} *, 1 \leq \mathrm{i} \leq \mathrm{n}$ y $\mathrm{x}_{1} \notin \mathrm{V}_{\mathrm{T}} *$, definimos la relación de derivación inmediata: 


$$
\left(\mathrm{x}_{1}, \ldots, \mathrm{x}_{\mathrm{n}}\right) \Rightarrow\left(\mathrm{y}_{1}, \ldots, \mathrm{y}_{\mathrm{n}}\right)
$$

si se da una de las siguientes situaciones:

(i) $\left|x_{i}\right|_{K}=0$, con $1 \leq i \leq n \wedge\left(\left(x_{i} \Rightarrow y_{i}\right) \vee\left(x_{i} \in V_{T} * \wedge x_{i}=y_{i}\right)\right.$, con $\left.1 \leq i \leq n\right)$, o bien

(ii) $\exists i, 1 \leq i \leq n$ : $\left|x_{i}\right|_{K}>0$; si $x_{i}=z_{1} Q_{i_{1}} z_{2} \ldots z_{m} Q_{i_{m}} z_{m+1}, z_{i} \in V_{\Gamma}^{*}, 1 \leq i \leq m+1$ y $\left|x_{i j}\right|_{K}=0$, para todo $\mathrm{j}$, con $1 \leq \mathrm{j} \leq \mathrm{m}$, entonces $\mathrm{y}_{\mathrm{i}}=\mathrm{z}_{1} \mathrm{xi}_{1} \mathrm{z}_{2} \ldots \mathrm{z}_{\mathrm{m}} \mathrm{xi}_{\mathrm{m}} \mathrm{z}_{\mathrm{m}+1}\left[\mathrm{e} \mathrm{yi}_{\mathrm{j}}=\mathrm{Si}_{\mathrm{j}}\right.$, con $1 \leq \mathrm{j} \leq \mathrm{m}$ ]; si $\left|\mathrm{xi}_{\mathrm{j}}\right|_{\mathrm{K}}>0$, para algún $\mathrm{j}, 1 \leq \mathrm{j} \leq \mathrm{m}$, entonces $\mathrm{y}_{\mathrm{i}}=\mathrm{x}_{\mathrm{i}}$; para todo $\mathrm{i}, 1 \leq \mathrm{i} \leq \mathrm{n}$, para el cual $\mathrm{y}_{\mathrm{i}}$ no esté especificado arriba, tendremos que $\mathrm{y}_{\mathrm{i}}=\mathrm{x}_{\mathrm{i}}$.

En otras palabras, de $\left(\mathrm{x}_{1}, \ldots, \mathrm{x}_{\mathrm{n}}\right)$ se deriva $\left(\mathrm{y}_{1}, \ldots, \mathrm{y}_{\mathrm{n}}\right)$ cuando:

(i) [paso de reescritura] no aparece en $\left(\mathrm{x}_{1}, \ldots, \mathrm{x}_{\mathrm{n}}\right)$ ningún símbolo de llamada: tenemos entonces una simple reescritura de los componentes, $x_{i} \Rightarrow_{i} y_{i}$, con $1 \leq i \leq n$, mediante la utilización de una regla para cada componente $\mathrm{P}_{\mathrm{i}}$, con excepción de los casos en que $\mathrm{x}_{\mathrm{i}}$ es terminal, en los que $\mathrm{y}_{\mathrm{i}}=\mathrm{x}_{\mathrm{i}}$, o bien

(ii) [paso de comunicación] aparece algún símbolo de llamada: toda ocurrencia de $Q_{j}$ en $x_{i}$ es sustituida por su forma sentencial $x_{j}$ siempre que $x_{j}$ no contenga a su vez símbolos de llamada.

Es importante tener presente que la comunicación tiene siempre prioridad sobre la reescritura.

El funcionamiento de un sistema de gramáticas PC se bloquea en dos casos:

(i) cuando un componente $x_{i}$ de la $n$-upla actual $\left(x_{1}, \ldots, x_{n}\right)$ no es terminal, pero no se le puede aplicar ninguna regla de $\mathrm{P}_{\mathrm{i}}$, o

(ii) cuando aparece una llamada circular: si $\mathrm{Pi}_{1}$ introduce $\mathrm{Qi}_{2}, \mathrm{Pi}_{2}$ introduce $\mathrm{Q}_{3}, \ldots$, $\mathrm{P}_{\mathrm{k}-1}$ introduce $\mathrm{Q}_{\mathrm{i}_{\mathrm{k}}}$, y $\mathrm{P}_{\mathrm{i}_{\mathrm{k}}}$ introduce $\mathrm{Q}_{1}$, no es posible la reescritura, porque la comunicación, según hemos dicho, tiene prioridad.

Definición 9. El lenguaje generado por un sistema de gramáticas $\mathrm{PC}$ :

$$
\Gamma=\left(\mathrm{V}_{\mathrm{N}}, \mathrm{K}, \mathrm{V}_{\mathrm{T}},\left(\mathrm{S}_{1}, \mathrm{P}_{1}\right), \ldots,\left(\mathrm{S}_{\mathrm{n}}, \mathrm{P}_{\mathrm{n}}\right)\right)
$$

se define como:

$$
\mathrm{L}(\Gamma)=\left\{\mathrm{x} \in \mathrm{V}_{\mathrm{T}} * \mid\left(\mathrm{S}_{1}, \ldots, \mathrm{S}_{\mathrm{n}}\right) \Rightarrow *\left(\mathrm{x}, \mathrm{y}_{2}, \ldots, \mathrm{y}_{\mathrm{n}}\right), \text { con } \mathrm{y}_{\mathrm{i}} \in \mathrm{V}_{\Gamma} *, 2 \leq \mathrm{i} \leq \mathrm{n}\right\} .
$$

Partimos, pues, de la n-upla de axiomas y avanzamos mediante sucesivos pasos de reescritura y comunicación hasta que el componente $\mathrm{P}_{1}$ produce una cadena terminal. Este componente es el director del sistema y su lenguaje es el de éste. Por tanto, mientras que en un sistema de gramáticas $\mathrm{CD}$ todos los componentes ocupan la misma posición,. en un sistema de gramáticas PC, en cambio, tenemos una jerarquía de dos niveles: el director del sistema y los que podemos llamar procesadores.

Hasta aquí hemos considerado sistemas de gramáticas PC en los que no se ha impuesto restricción alguna sobre el uso de los símbolos de llamada, es decir, cualquier componente $\mathrm{P}_{\mathrm{i}}$ puede introducir cualquier símbolo $\mathrm{Q}_{\mathrm{j}}$.

Definición 10. Sea un sistema de gramáticas PC: 


$$
\Gamma=\left(\mathrm{V}_{\mathrm{N}}, \mathrm{K}, \mathrm{V}_{\mathrm{T}},\left(\mathrm{P}_{1}, \mathrm{~S}_{1}\right), \ldots,\left(\mathrm{P}_{\mathrm{n}}, \mathrm{S}_{\mathrm{n}}\right)\right) .
$$

Si únicamente $\mathrm{P}_{1}$ puede introducir símbolos de llamada, esto es, si:

$$
\mathrm{P}_{\mathrm{i}} \subseteq\left(\mathrm{V}_{\mathrm{N}} \cup \mathrm{V}_{\mathrm{T}}\right)^{*} \times\left(\mathrm{V}_{\mathrm{N}} \cup \mathrm{V}_{\mathrm{T}}\right)^{*} \text {, }
$$

para $2 \leq \mathrm{i} \leq \mathrm{n}$, entonces decimos que $\Gamma$ es un sistema centralizado. En caso contrario, es un sistema no-centralizado.

Definición 11. Un sistema de gramáticas PC es un sistema retornante si, después de la comunicación, todo componente una cadena del cual se ha enviado a otro componente vuelve a su axioma. Si eliminamos la expresión de la definición 8 que hemos colocado entre corchetes, [e $\mathrm{yi}_{\mathrm{j}}=\mathrm{Si}_{\mathrm{j}}$, con $1 \leq \mathrm{j} \leq \mathrm{m}$ ], obtenemos un sistema no-retornante: después de la comunicación, el componente $\mathrm{P}_{\mathrm{i}_{\mathrm{j}}}$ no vuelve a $\mathrm{S}_{\mathrm{j}}$, sino que sólo ha enviado una copia de la cadena y continúa procesando la original en el estado en que se encuentra. Así pues, para un sistema PC $\Gamma$, tendremos un lenguaje en el modo retornante $\mathrm{L}_{\mathrm{r}}(\Gamma)$ (obtenido mediante un modo de derivación que notamos como $\Rightarrow_{\mathrm{r}}$ ) y un lenguaje en el modo no-retornante $\mathrm{L}_{\mathrm{nr}}(\Gamma)$ (obtenido mediante un modo de derivación que notamos como $\Rightarrow_{\mathrm{nr}}$ ).

Para ilustrar las definiciones, vamos a escoger a continuación un par de sistemas que ofrecen interpretaciones lingüísticamente relevantes: son conocidos lenguajes noindependientes del contexto que corresponden a ciertos fenómenos que encontramos en el lenguaje natural.

Ejemplo 12. Sea el siguiente sistema de gramáticas PC:

$$
\Gamma=\left(\left\{S_{1}, S_{2}, S_{3}\right\}, K,\{a, b, c\},\left(S_{1}, P_{1}\right),\left(S_{2}, P_{2}\right),\left(S_{3}, P_{3}\right)\right),
$$

con:

$$
\begin{aligned}
\mathrm{P}_{1}=\left\{\mathrm{S}_{1} \rightarrow a S_{1}, \mathrm{~S}_{1}\right. & \left.\rightarrow \mathrm{a}^{3} \mathrm{Q}_{2}, \mathrm{~S}_{2} \rightarrow \mathrm{b}^{2} \mathrm{Q}_{3}, \mathrm{~S}_{3} \rightarrow \mathrm{c}\right\}, \\
\mathrm{P}_{2} & =\left\{\mathrm{S}_{2} \rightarrow b S_{2}\right\}, \\
\mathrm{P}_{3} & =\left\{\mathrm{S}_{3} \rightarrow \mathrm{cS}_{3}\right\} .
\end{aligned}
$$

Obtenemos el siguiente lenguaje:

$$
\mathrm{L}_{\mathrm{r}}(\Gamma)=\mathrm{L}_{\mathrm{nr}}(\Gamma)=\left\{\mathrm{a}^{\mathrm{n}} \mathrm{b}^{\mathrm{n}} \mathrm{c}^{\mathrm{n}} \mid \mathrm{n} \geq 1\right\} .
$$

Esta expresión formaliza el fenómeno lingüístico conocido como concordancias múltiples.

Examinemos brevemente cómo funciona $\Gamma$. Empezamos con $\left(\mathrm{S}_{1}, \mathrm{~S}_{2}, \mathrm{~S}_{3}\right)$. Utilizando la primera regla de $\mathrm{P}_{1}$ y las únicas que forman parte de $\mathrm{P}_{2}$ y $\mathrm{P}_{3}$ para $n \geq 0$ pasos, tenemos que:

$$
\left(\mathrm{S}_{1}, \mathrm{~S}_{2}, \mathrm{~S}_{3}\right) \Rightarrow_{\mathrm{r}}\left(\mathrm{a}^{\mathrm{n}} \mathrm{S}_{1}, \mathrm{~b}^{\mathrm{n}} \mathrm{S}_{2}, \mathrm{c}^{\mathrm{n}} \mathrm{S}_{3}\right)
$$

Tomamos ahora la segunda regla de $\mathrm{P}_{1}$ :

$$
\left(a^{n} S_{1}, b^{n} S_{2}, c^{n} S_{3}\right) \Rightarrow_{r}\left(a^{n+3} Q_{2}, b^{n+1} S_{2}, c^{n+1} S_{3}\right) .
$$

Como ha aparecido el símbolo de llamada $\mathrm{Q}_{2}$, hemos de ejecutar un paso de comunicación. Así pues, enviamos $b^{n+1} S_{2}$ al primer componente para sustituir a $\mathrm{Q}_{2}$ :

$$
\left(a^{n+3} Q_{2}, b^{n+1} S_{2}, c^{n+1} S_{3}\right) \Rightarrow_{r}\left(a^{n+3} b^{n+1} S_{2}, S_{2}, c^{n+1} S_{3}\right)
$$


Continuamos de la siguiente manera:

$$
\begin{aligned}
& \left(a^{n+3} b^{n+1} S_{2}, S_{2}, c^{n+1} S_{3}\right) \Rightarrow{ }_{r}\left(a^{n+3} b^{n+3} Q_{3}, b S_{2}, c^{n+2} S_{3}\right) \Rightarrow_{r}\left(a^{n+3} b^{n+3} c^{n+2} S_{3}, b S_{2}, S_{3}\right) \Rightarrow_{r} \\
& \left(a^{n+3} b^{n+3} c^{n+3}, b^{2} S_{2}, c S_{3}\right) \text {. }
\end{aligned}
$$

Como hay una única llamada de $\mathrm{P}_{1}$ a $\mathrm{P}_{2}$ y a $\mathrm{P}_{3}$, en $\Gamma$ los modos retornante y noretornante coinciden.

Ejemplo 13. Sea el siguiente sistema de gramáticas PC:

$$
\Gamma=\left(\left\{\mathrm{S}_{1}, \mathrm{~S}_{2}\right\}, \mathrm{K},\{\mathrm{a}, \mathrm{b}\},\left(\mathrm{S}_{1}, \mathrm{P}_{1}\right),\left(\mathrm{S}_{2}, \mathrm{P}_{2}\right)\right),
$$

con:

$$
\begin{gathered}
P_{1}=\left\{S_{1} \rightarrow S_{1}, S_{1} \rightarrow Q_{2} Q_{2}\right\}, \\
P_{2}=\left\{S_{2} \rightarrow a S_{2}, S_{2} \rightarrow b S_{2}, S_{2} \rightarrow a, S_{2} \rightarrow b\right\} .
\end{gathered}
$$

Obtenemos el lenguaje:

$$
\mathrm{L}_{\mathrm{r}}(\Gamma)=\mathrm{L}_{\mathrm{nr}}(\Gamma)=\left\{\mathrm{xx} \mid \mathrm{x} \in\{\mathrm{a}, \mathrm{b}\}^{+}\right\} .
$$

Esta expresión formaliza el fenómeno lingüístico conocido como reduplicación.

El funcionamiento de $\Gamma$ es sencillo. $\mathrm{P}_{1}$ permanece inactivo y entretanto $\mathrm{P}_{2}$ genera una cadena. Luego $P_{1}$ introduce $Q_{2} Q_{2}$, con lo que la cadena generada por $P_{2}$ es enviada a $P_{1}$ y duplicada (ha de ser terminal; si no, el sistema queda bloqueado).

Obsérvese que los sistemas de los ejemplos 12 y 13 son centralizados.

Veamos ahora un caso en el que los lenguajes retornante y no-retornante no coinciden.

Ejemplo 14. Sea el siguiente sistema de gramáticas PC:

$$
\Gamma=\left(\left\{\mathrm{S}_{1}, \mathrm{~S}_{2}\right\}, \mathrm{K},\{\mathrm{a}\},\left(\mathrm{S}_{1}, \mathrm{P}_{1}\right),\left(\mathrm{S}_{2}, \mathrm{P}_{2}\right)\right\},
$$

con:

$$
\begin{gathered}
P_{1}=\left\{S_{1} \rightarrow a Q_{2}, S_{2} \rightarrow a Q_{2}, S_{2} \rightarrow a\right\}, \\
P_{2}=\left\{S_{2} \rightarrow a S_{2}\right\} .
\end{gathered}
$$

Obtenemos los siguientes lenguajes:

$$
\begin{gathered}
\mathrm{L}_{\mathrm{r}}(\Gamma)=\left\{\mathrm{a}^{2 \mathrm{n}+1} \mid \mathrm{n} \geq 1\right\}, \\
\mathrm{L}_{\mathrm{nr}}(\Gamma)=\left\{\mathrm{a}^{(\mathrm{n}+1)(\mathrm{n}+2) / 2} \mid \mathrm{n} \geq 1\right\} .
\end{gathered}
$$

\section{Capacidad generativa y complejidad descriptiva}

Las reglas que hemos empleado en el ejemplo 12 son todas ellas regulares, y las del ejemplo 13 son algunas de ellas regulares y otras independientes del contexto. En ambos casos, utilizadas aisladamente, sin que formen parte de un sistema de gramáticas, no pueden generar -como es bien sabido- las estructuras lingüísticas mencionadas, que son noindependientes del contexto. En cambio, cuando tales reglas se integran en un sistema y funcionan coordinadamente de acuerdo con el protocolo de cooperación, pueden generar con una gran simplicidad las estructuras deseadas. Vemos, así, que conjuntos de reglas simples y de poca capacidad generativa, si son organizados de manera adecuada en forma de sistema, alcanzan una potencia inusual en la teoría clásica de lenguajes formales. 
Disponemos, además, de algunos resultados significativos sobre la complejidad descriptiva de los dos tipos de sistemas de gramáticas, especialmente en comparación con las gramáticas independientes del contexto. Así, por ejemplo, para las medidas de complejidad Var (número de elementos no-terminales), Prod (número de producciones) y Symb (número de símbolos que se utilizan en las producciones), y para una buena cantidad de sistemas $\mathrm{CD}$ y $\mathrm{PC}$, comprobamos que hay a menudo lenguajes independientes del contexto que se pueden generar de una manera mucho más económica con sistemas que con gramáticas aisladas. En muchos casos obtenemos resultados del estilo siguiente: existe una secuencia $L_{n}$, con $n \geq 1$, de lenguajes independientes del contexto tal que $M_{C F}\left(L_{n}\right) \geq n$, para $\mathrm{n} \geq 1$, pero $\mathrm{M}_{\mathrm{PC}}\left(\mathrm{L}_{\mathrm{n}}\right) \leq \mathrm{p}$, donde $\mathrm{p}$ es una constante y $\mathrm{M}$ una de las medidas de complejidad descriptiva.

Los sistemas de gramáticas están empezando a ser aplicados a la descripción de las lenguas naturales. Buenas muestras de ello son Csuhaj-Varjú 1994 y Jiménez López 1996. 


\section{REFERENCIAS}

E. Csuhaj-Varjú (1994), “Grammar systems: a multi-agent framework for natural language generation", en Gh. Păun, ed., Mathematical aspects of natural and formal languages: 63-78. World Scientific, Singapore.

E. Csuhaj-Varjú \& J. Dassow (1989), “On cooperating distributed grammar systems”, Journal of Information Processing and Cybernetics (EIK), 26, 1-2: 49-63.

E. Csuhaj-Varjú, J. Dassow, J. Kelemen \& Gh. Păun (1994), Grammar systems: a grammatical approach to distribution and cooperation. Gordon and Breach, London.

M. D. Jiménez López (1996), "Sistemas de gramáticas y lenguajes naturales: ideas intuitivas al respecto", en C. Martín Vide, ed., Lenguajes naturales y lenguajes formales, XII: 223-236. PPU, Barcelona.

R. Meersman \& G. Rozenberg (1978), “Cooperating grammar systems”, en Lecture Notes in Computer Science, vol. 64: 364-373. Springer, Berlin.

Gh. Păun (1995a), "Generating languages in a distributed way: grammar systems", en C. Martín Vide, ed., Lenguajes naturales y lenguajes formales, XI: 45-67. PPU, Barcelona.

Gh. Păun (1995b), "Grammar systems: a grammatical approach to distribution and cooperation”, en Z. Fulop \& F. Gecseg, eds., Lecture Notes in Computer Science, vol. 944: 429-443. Springer, Berlin.

Gh. Păun (1995c), "Parallel communicating grammar systems. A survey”, en C. Martín Vide, ed., Lenguajes naturales y lenguajes formales, XI: 257-283. PPU, Barcelona.

Gh. Păun \& L. Sântean (1989), "Parallel communicating grammar systems: the regular case", Annals of the University of Bucharest: Series Mathematics-Informatics, 38: 55-63.

A. Salomaa (1973), Formal languages. Academic Press, New York. 
